\title{
Cell interactions with the extracellular matrix
}

\author{
L. Bruckner-Tuderman • K. von der Mark • \\ T. Pihlajaniemi $\cdot$ K. Unsicker
}

Published online: 10 November 2009

(C) Springer-Verlag 2009

\section{Introduction}

The idea of a special issue on cell interactions with the extracellular matrix (ECM) is a result of the increasing awareness about the integral functions of the ECM in virtually all dynamic processes of development, maintenance, reconstruction and disease. The purpose of this special issue is not to present a comprehensive overview of all macromolecules that form the ECM and their interacting cellular receptors, as many excellent reviews and monographs have been published recently on this topic (Myllyharju and Kivirikko 2004; Kadler et al. 2007; Bateman et al. 2009). Rather, it is the guest editors' objective to present recent advances in ECM-cell interactions as seen from the perspective of dynamic cell performances during development and in various disease states.

\section{K. Unsicker $(\bowtie)$}

Department of Molecular Embryology,

Institute of Anatomy and Cell Biology,

University of Freiburg, Albertstrasse 17,

79104 Freiburg, Germany

e-mail: ku39@anat.uni-freiburg.de

L. Bruckner-Tuderman

Department of Dermatology,

University Medical Center Freiburg, Hauptstrasse 7,

79104 Freiburg, Germany

K. von der Mark

Nikolaus Fiebiger Centre of Molecular Medicine,

University of Erlangen-Nuernberg, Glückstrasse 6,

91054 Erlangen, Germany

T. Pihlajaniemi

Oulu Center for Cell-Matrix Biology,

Biocenter and Department of Medical Biochemistry

and Molecular Biology,

University of Oulu, P.O. Box 5000, Aapistie 7,

90014 Oulu, Finland
The importance of ECM biology and pathology is immediately recognised when thinking of the extent to which ECM molecules and their cellular receptors are involved in diseases. A wide range of diseases, initially not understood under the aspect of ECM pathology, exists in which the ECM contributes considerably to onset and progression. Among these are all fibrotic diseases of the skin, liver, lung and kidney, some skeletal, muscular and neurodegenerative diseases and diseases in clinical fields, such as pulmology, cardiology, and ophthalmology. Only the comprehensive analysis of ECM genes has provided insights into genotypephenotype relationships of hereditary connective tissue diseases such as osteogenesis imperfecta, numerous skeletal displasias, Ehlers-Danlos syndrome, Marfan syndrome and epidermolysis bullosa. Failures in wound healing, fracture repair and degenerative autoimmune diseases critically involve disregulation of ECM metabolism or autoimmune reactions against matrix macromolecules.

In addition to diseases with a crucial involvement of the ECM, physiological processes, such as stem cell differentiation, which have previously not been seen in the context of ECM involvement, are now increasingly acknowledged as being crucially dependent on the composition of the ECM and the stromal cells.

\section{A brief overview of articles in this special issue}

The series of articles on cell interactions with the ECM begins with a presentation by Bruckner (2009) of the hierarchical organisation of the ECM and the assembly of macromolecules into insoluble suprastructural elements. Such suprastructural elements can be fibrils, microfibrils or networks, which, in turn, are assembled into regional tissue structures, such as fibres or basement membranes. Interestingly, most, if not all ECM suprastructures are co- 
polymers of more than one molecular species that differ in their identity and relative abundance. Thus, ECM suprastructures are composite biological amalgamates. In spite of this, most studies in matrix biology have focused so far on molecular features and mechanisms. The article describes recent progress that has been made in identifying principles of interactions within suprastructural elements and their functional consequences.

The article by Myllyharju and Schipani (2009) introduces ECM genes as hypoxia-inducible targets. The ability of cells to survive under hypoxia or adapt to it depends on a family of hypoxia-inducible transcription factors (HIFs) that induce the expression of a number of genes involved in haematopoiesis, angiogenesis, iron transport, glucose utilisation, resistance to oxidative stress, cell proliferation, survival and apoptosis and ECM homeostasis. The authors describe the recently identified molecular mechanisms responsible for the oxygen-dependent stability and discuss the vital role of the hypoxia response pathway in chondrogenesis and joint development.

Xian et al. (2009) describe syndecans as receptors and organisers of the ECM. Syndecans are type I transmembrane proteins having a core protein modified with glycosaminoglycan (GAG) chains, most commonly heparan sulphate. A large number of molecules can interact with heparan sulphate, most notably collagens and glycoproteins of the ECM. Frequently, they do so in conjunction with other receptors, e.g. integrins. Syndecans can interact with actinassociated proteins and signalling molecules such as protein kinases. Evidence from syndecan knockout mice suggests that syndecans have roles in postnatal tissue repair, inflammation and tumour progression.

The role of the ECM in lymphocyte migration is discussed by Korpos et al. (2009). The ECM can act either as a barrier to migrating leukocytes (basement membranes) or provide a physical scaffold supporting or guiding migration (interstitial matrix). This review focuses on basement membranes and current knowledge of the way that leukocytes transmigrate basement membranes, with emphasis on $\mathrm{T}$ lymphocytes. Increasing evidence suggests that the classical concept of cell-matrix adhesion may require revision with respect to leukocyte-ECM interactions. Whereas specific receptors may be required for the leukocyte recognition of ECM molecules or three-dimensional structural domains, the role of adhesion in migration is less clear. Indirect effects of the ECM such as the binding and presentation of cytokines or chemotactic factors may more profoundly influence the directed migration of normally non-adherent leukocytes than the migration of adherent cells such as epithelial cells or fibroblasts. Proteases released at sites of inflammation process specific chemokines and selectively cleave ECM, cell surface molecules or soluble factors; this might result in the release of bioactive fragments that can function as chemoattractants for various leukocyte subsets or may modulate the activity/function of resident mesenchymal and immune cells. Current data suggest that different leukocyte types employ different mechanisms to migrate across or through the ECM and that this is determined by the composition and organisation of the ECM.

Classical Osteogenesis imperfecta (OI) is a dominant genetic disorder of connective tissue caused by mutations in either of the two genes encoding type I collagen, COL1A1 and COL1A2. Recent investigations, however, have generated a new paradigm for OI that incorporates many of the prototypical features that distinguish dominant and recessive conditions within a type I collagen framework. Marini et al. (2009) present evidence from their own and other laboratories showing that the long-sought cause of the recessive form of OI first postulated in the Sillence classification is a range of defects in the genes encoding cartilage-associated protein (CRTAP) or prolyl 3-hydroxylase 1 (P3H1/LEPRE1). Together with cyclophilin B (PPIB), CRTAP and P3H1 comprise the collagen prolyl 3-hydroxylation complex, which catalyzes a specific posttranslational modification of types I, II and V collagens and may act as a general chaperone. Patients with mutations in CRTAP or LEPRE1 have a lethal to severe osteochondrodystrophy that overlaps with Sillence types II and III OI but that has distinctive features.

The article by Ramirez and Sakai (2009) concentrates on fibrillins, which are large cysteine-rich glycoproteins serving as supporting structures that impart tissue integrity and as regulators of signalling events that instruct cell performance. Fibrillins exert their structural role through the temporal and hierarchical assembly of microfibrils and elastic fibres, whereas the instructive role reflects the ability of fibrillins to sequester transforming growth factor $\beta$ (TGF $\beta$ ) and bone morphogenetic protein (BMP) complexes in the ECM. Characterisation of fibrillin mutations in human patients and genetically engineered mice has demonstrated that perturbation of either function manifests in disease. These studies have also indicated that fibrillins are integral components of a broader biological network of extracellular, cell surface and signalling molecules that orchestrate morphogenetic and homeostatic programs in multiple organ systems. Moreover, these analyses have suggested that the relative composition of fibrillin-rich microfibrils imparts contextual specificity to TGF $\beta$ and BMP signalling by concentrating the ligands locally so as to regulate cell differentiation within a spatial context during organ formation (positive regulation) and by restricting their bioavailability so as to modulate cell performance in a timely fashion during tissue remodelling/ repair (negative regulation). The emerging view is that fibrillin-rich microfibrils are molecular integrators of structural and instructive signals with TGF $\beta$ and BMPs.

The article by Schmidt and Friedl (2009) addresses the role of different adhesion systems, as e.g. integrins, syndecans, CD44, and discoidin domain receptors (DDRs), 
which together coordinate ligand binding with direct or indirect cytoskeletal coupling and intracellular signalling, in cell migration. He describes 2D and 3D migration models and discusses the hierarchy of these concurrent adhesion systems, their specific tasks in cell migration, and how they contribute to migration in three-dimensional, multi-ligand tissue environments.

The article by Tsang et al. (2009) highlights the developmental roles of the ECM. Elucidation of the role that the ECM plays in developmental processes has been significantly advanced through studying the phenotypic and developmental consequences of specific genetic alterations of ECM components in the mouse. These studies have revealed the enormous contribution of the ECM to regulating key processes in morphogenesis and organogenesis such as cell adhesion, proliferation, specification, migration, survival and differentiation. The ECM interacts with signalling molecules and morphogens thereby modulating their activities and underscoring their signalling capacities.

In their article, Ivaska and Heino (2009) focus on the interplay between cell adhesion and growth factor receptors. All metazoans express integrin-type adhesion receptors and receptors for growth factors. Integrins recognise ECM proteins and, after ligand binding, can activate the same cellular signalling pathways that are regulated by growth factor receptors. The authors review the present information about the role of integrins as assisting growth factor receptors and the interplay between the receptors in cell signalling and in the orchestration of receptor recycling.

Integrin-mediated cell adhesion to the ECM plays an important role in regulating stem cell function and maintenance. This is the subject of the article by Ellis and Tanentzapf (2009). Integrins help define and shape the microenvironment in which stem cells are found, viz. the stem cell niche. Integrins have diverse roles with respect to stem cells: homing to their niche, maintaining them in the niche, developing niche architecture, regulating stem cell proliferation and self renewal and, finally, controlling the orientation of dividing stem cells.

Cues from the ECM for nanoscale engineering of biomimetic surfaces are addressed by von der Mark et al. (2009). The article highlights the importance of the nanoscale structure of extracellular matrices and the way that information on the structure, biomechanical properties and adhesiveness of ECM proteins and their interactions with cellular receptors have been utilised in the design of biomimetic scaffolds and surfaces for tissue engineering. Recent approaches are described with regard to achieving adhesiveness and bioactivity of biomaterials based on coatings with ECM macromolecules such as fibronectin, laminin and collagens, their integrin binding epitopes RGD or the collagen-derived triplehelical peptide GFOGER. The article points out that this nanotechnology not only has contributed to our understanding of the molecular mechanism of cell-matrix interactions by generating geometrically defined adhesive surface patterns at the nanoscale, but also has introduced new biomimetic materials with physically, biologically and chemically mediated surface modifications that control cell functions including adhesion, growth, migration, differentiation and apoptosis specifically and solely on the basis of their nanoscale surface pattern

In their article, Nicolae and Olsen (2009) discuss three genetic disorders in which major pathogenetic features have been traced back to alterations in the levels/activities of ECM components. In each case, disease-associated alterations are found both intra- and extracellularly. Cherubism is a case of inflammatory bone loss in the jaws of children. The primary defect involves an intracellular signalling molecule, although a major pathogenetic component and therapeutic target of the disease is the extracellular cytokine tumor necrosis factor alpha. Knobloch syndrome is caused by recessive mutations in collagen XVIII. Although this protein has been classified as belonging to a group of structural macromolecules, the consequence of the mutations is impairment of cellular metabolism. The third disorder, infantile haemangioma, is a common tumour of capillary endothelial cells in infancy. The tumour appears within a few days/weeks after birth, grows rapidly over several months and regresses over several years.

Basement membrane involvement in human disease is the topic of the article by van Agtmael and BrucknerTuderman (2009). Since the first documentation of a role of basement membranes in human disease (the COL $4 A 5$ mutations in Alport's syndrome), the number of diseases caused by mutations in basement membrane components has steadily increased. However, many questions remain as to the molecular and cellular consequences of these mutations and the way that they lead to the observed disease phenotypes. Despite this, exciting progress has recently been made with potential treatment options for some of these so far incurable diseases.

$R U N X 2$ is a multifunctional transcription factor that controls skeletal development by regulating the differentiation of chondrocytes and osteoblasts. Komori (2009) describes the role that it plays at the late stage of chondrocyte differentiation: RUNX2 is required for chondrocyte maturation and regulates Colloal expression in hypertrophic chondrocytes and the expression of Spp1, Ibsp and Mmp13 in terminal hypertrophic chondrocytes. RUNX2 commits pluripotent mesenchymal cells to the osteoblast lineage. During osteoblast differentiation, RUNX2 upregulates the expression of bone matrix protein genes including Col1a1, Spp1, Ibsp, Bglap and Fn1 in vitro. However, overexpression of Runx 2 inhibits osteoblast maturation and reduces Colla1 and Bglap expression. 
During bone development, RUNX2 induces osteoblast differentiation and increases the number of immature osteoblasts, which form immature bone, whereas Runx2 expression needs to be downregulated for differentiation into mature osteoblasts. Similarly, RUNX2 inhibits terminal differentiation of odontoblasts.

In his article, Boot-Handford (2009) describes the unfolded protein response and its relevance to connective tissue diseases. The unfolded protein response has evolved to counter the stresses that occur in the endoplasmic reticulum as a result of misfolded proteins. This sophisticated quality control system attempts to restore homeostasis through the action of a number of different pathways that are coordinated in the first instance by the endoplasmic reticulum (ER) stress-senor proteins IRE1, ATF6 and PERK. Fibroblasts, osteoblasts and chondrocytes synthesise and secrete large quantities of proteins. Mutations in many of these gene products give rise to heritable connective tissue disorders. Until recently, these mutant gene products were thought to exert their effect through the assembly of a defective ECM. However, it is now becoming clear that ER stress and the unfolded protein response, because of the expression of a mutant gene product, is not only a feature of the initiation and progression of a whole range of different connective tissue diseases, but may also be a key mediator in these processes.

Antibodies against cartilage proteins are highly prevalent in the sera and synovial fluids of rheumatoid arthritis (RA) patients and also precede disease induction in various spontaneous and induced animal models of arthritis. Nandakumar (2009) describes the role played by these antibodies in the induction and perpetuation of disease and underlying mechanisms. Antibodies binding to cartilage protein(s), especially the major articular cartilage protein, collagen type II (CII), can induce, in naive mice, an acute form of arthritis that can substantially destroy the cartilage and bone architecture. More importantly, these anti-CII antibodies can also directly cause the destruction of the target tissue preceding and independent of disease development. Antibodies to citrullinated protein antigens and rheumatoid factor are well-validated prognostic and diagnostic markers of severe erosive RA. Nandakumar's group has recently found that monoclonal antibodies to citrullinemodified cartilage protein can bind cartilage and synovial tissue and mediate arthritis in mice. Interestingly, recent findings have also showed that enzymatic cleavage or modification of pathogenic IgG antibodies protect the cartilage surface opening up new therapeutic possibilities for protecting the cartilage from inflammatory damage.

Cartilage, bone, tendon and ligaments of joints all consist of an ECM made of collagens, proteoglycans and specialised glycoproteins that are actively synthesised, precisely assembled and subsequently degraded by resident connective tissue cells. Cawston and Young (2009) describe the role of different classes of proteinases in connective tissue turnover. Metalloproteinases (MMPs) are potent enzymes that, once activated, degrade connective tissue but are inhibited by tissue inhibitors of metalloproteinases (TIMPs); the balance between active MMPs and TIMPs determines, in many tissues, the extent of ECM degradation. The serine proteinases are involved in the initiation of activation cascades and some, such as elastase, can directly degrade the matrix. Cysteine proteinases are responsible for the breakdown of collagen in bone. Various growth factors increase the synthesis of matrix and proteinase inhibitors, whereas cytokines, alone or in combination, can inhibit matrix synthesis and stimulate proteinase production and matrix destruction.

A final series of four articles present brief overviews of key ECM protein families, namely proteoglycans, collagens and laminins and the major ECM receptor family, the integrins.

Schaefer and Schaefer (2009) present recent discoveries on proteoglycans, which can be classified into three major categories: (1) small leucine-rich proteoglycans (SLRPs), (2) modular proteoglycans and (3) cell surface proteoglycans. Proteoglycans not only are structural proteins, but also play major roles in signal transduction with regulatory functions in various cellular processes. They can modulate distinct pathways, including those driven by BMP/TGF superfamily members, receptor tyrosine kinases, the insulin-like growth factor-I receptor, and Toll-like receptors.

Gordon and Hahn (2009) review collagen family members, important aspects of their structure and functions and associated diseases. Collagens are ECM molecules used by cells for structural integrity and a variety of other functions. Alpha chains are used to build trimeric molecules, which are woven together into a triple helix in at least one region. The triple helical domains form as a result of glycine (gly) being used every third residue (i.e. repeating peptide triplets of gly$\mathrm{X}-\mathrm{Y}$ ). In this triplet, $\mathrm{X}$ is often proline and $\mathrm{Y}$ is often hydroxyproline. In each $\alpha$ chain, triple helical regions ( $\mathrm{Col}$ domains) are flanked by non-collagenous (non-gly-X-Y) regions (NC domains). These $\mathrm{NC}$ domains often contain recognisable peptide modules found in other matrix molecules. The function of a collagen often depends on the proper supramolecular assembly of molecules into an aggregate that becomes incorporated into the matrix. The review highlights some of the structural characteristics of the numbered collagens, types I-XXVIII, but does not include the growing number of triple helix-containing molecules that are not among the numbered collagens, many of which (e.g. the collectin family) are involved in innate immunity.

Laminins, described by Durbeej (2009), comprise a family of cell adhesion glycoproteins found predominantly in basement membranes. Many laminins self-assemble to form networks that remain in close association with cells through interactions with cell surface receptors. Laminins are essential 
for early embryonic development and organogenesis and have crucial functions in many tissues. This "at-a-glance" article gives a snapshot of laminin structure, tissue distribution and interactions with other matrix molecules and receptors and briefly describes laminin mutations in mice and humans.

Barczyk et al. (2009) summarise some basic aspects of the integrin family including their history, structure and ligandbinding properties. Integrins play important roles during developmental and pathological processes. The family consists of $24 \alpha \beta$ heterodimeric members that mediate the attachment of cells to the ECM but which also take part in specialised cell-cell interactions. Only a subset of integrins (eight out of 24) recognises the RGD sequence in native ligands. In some ECM molecules, such as collagen and certain laminin isoforms, the RGD sequences are exposed upon denaturation or proteolytic cleavage, allowing cells to bind these ligands by using RGD-binding receptors. Proteolytic cleavage of ECM proteins might also generate fragments with novel biological activity, such as endostatin, tumstatin and endorepellin. Nine integrin chains contain an $\alpha$ I domain, including the collagen-binding integrins $\alpha 1 \beta 1$, $\alpha 2 \beta 1, \alpha 10 \beta 1$ and $\alpha 11 \beta 1$. The collagen-binding integrins recognise the triple-helical GFOGER sequence in the major collagens but their ability to recognise these sequences in vivo is dependent on the fibrillar status and accessibility of the interactive domains in the fibrillar collagens.

\section{References}

Barczyk M, Carracedo S, Gullberg D (2009) Integrins at a glance. Cell Tissue Res. doi:10.1007/s00441-009-0834-6

Bateman JF, Boot-Handford RP, Lamandé SR (2009) Genetic diseases of connective tissues: cellular and extracellular effects of ECM mutations. Nat Rev Genet 10:173-183

Boot-Handford RP (2009) The unfolded protein response and its relevance to connective tissue diseases. Cell Tissue Res. doi: 10.1007/s00441-009-0877-8

Bruckner P (2009) Suprastructures of extracellular matrices: Paradigms of functions controlled by aggregates rather than molecules. Cell Tissue Res. doi:10.1007/s00441-009-0864-0

Cawston T, Young D (2009) Proteinases involved in matrix turnover during cartilage and bone breakdown. Cell Tissue Res. doi:10.1007/s00441-009-0887-6
Durbeej M (2009) Laminins. Cell Tissue Res. doi:10.1007/s00441009-0838-2

Ellis S, Tanentzapf G (2009) Integrin-mediated adhesion and stem cell-niche interactions. Cell Tissue Res. doi:10.1007/s00441-0090828-4

Gordon M, Hahn R (2009) Collagens. Cell Tissue Res. doi:10.1007/ s00441-009-0844-4

Ivaska J, Heino J (2009) Interplay between cell adhesion and growth factor receptors: from the plasma membrane to the endosomes. Cell Tissue Res. doi:10.1007/s00441-009-0857-z

Kadler KE, Baldock C, Bella J, Boot-Handford RP (2007) Collagens at a glance. J Cell Sci 120:1955-1958

Komori T (2009) Regulation of bone development and extracellular matrix protein genes by RUNX2. Cell Tissue Res. doi:10.1007/ s00441-009-0832-8

Korpos E, Wu C, Song J, Hallmann R, Sorokin L (2009) Role of the extracellular matrix in lymphocyte migration. Cell Tissue Res. doi:10.1007/s00441-009-0853-3

Marini J, Cabral W, Barnes A (2009) Null mutations in LEPRE1 and CRTAP cause severe recessive osteogenesis imperfecta. Cell Tissue Res. doi:10.1007/s00441-009-0872-0

Mark K von der, Park J, Bauer S, Schmuki P (2009) Nanoscale engineering of biomimetic surfaces: cues from the ECM. Cell Tissue Res. doi:10.1007/s00441-009-0896-5

Myllyharju J, Kivirikko KI (2004) Collagens, modifying enzymes and their mutations in humans, flies and worms. Trends Genet 20: $33-43$

Myllyharju J, Schipani E (2009) Extracellular matrix genes as hypoxia-inducible targets. Cell Tissue Res. doi:10.1007/s00441009-0841-7

Nandakumar KS (2009) Pathogenic antibody recognition of cartilage. Cell Tissue Res. doi:10.1007/s00441-009-0816-8

Nicolae C, Olsen BR (2009) Unexpected matrix diseases and novel therapeutic strategies. Cell Tissue Res. doi:10.1007/s00441-0090874-y

Ramirez F, Sakai L (2009) Biogenesis and function of fibrillin assemblies. Cell Tissue Res. doi:10.1007/s00441-009-0822-x

Schaefer L, Schaefer R (2009) Proteoglycans: from structural compounds to signaling molecules. Cell Tissue Res. doi:10. 1007/s00441-009-0821-y

Schmidt S, Friedl P (2009) Interstitial cell migration: integrindependent and alternative adhesion mechanisms. Cell Tissue Res. doi:10.1007/s00441-009-0892-9

Tsang K, Cheung M, Chan D, Cheah K (2009) The developmental roles of the extracellular matrix - beyond structure to regulation. Cell Tissue Res. doi:10.1007/s00441-009-0893-8

Van Agtmael T, Bruckner-Tuderman L (2009) Basement membranes and human disease. Cell Tissue Res. doi:10.1007/s00441-0090866-y

Xian X, Gopal S, Couchman J (2009) Syndecans as receptors and organisers of the extracellular matrix. Cell Tissue Res. doi:10. 1007/s00441-009-0829-3 